\title{
Toxicovigilance I: A survey of acute poisonings in South Africa based on Tygerberg Poison Information Centre data
}

\author{
D J H Veale, Dip Pharm, BSc Hons, MSc, PhD; C A Wium, BSc, BSc Hons, MSc; G J Müller BSc, BSc Hons, MB ChB, MMed, PhD
}

Tygerberg Poison Information Centre, Department of Medicine, Faculty of Health Sciences, Stellenbosch University and Tygerberg Academic Hospital, Parow, Cape Town

Corresponding author: D J H Veale (djhveale@gmail.com)

\begin{abstract}
Background. The incidence and spectrum of acute poisonings in South Africa are unknown. Poisoning data can be derived from sources such as hospital admission records and poison information centre (PIC) records.

Objectives. This study was conducted to examine the extent of the problem and to identify trends and toxicovigilance issues using PIC data. Methods. A survey was conducted based on Tygerberg Poison Information Centre (TPIC) consultations over 1 year. TPIC consultation forms were analysed for patient demographics and causes of poisoning.

Results. The TPIC dealt with 4771 consultations related to human exposures to poisonous substances. The study showed that accidental exposure was more common than intentional poisoning ( $65.2 \%$ v. $34.8 \%$ ); that $55.8 \%$ of cases were adults, of which $57.6 \%$ were females; and that $61.4 \%$ of adult cases were intentional exposures, and of these $64.3 \%$ were females. There was a predominance of accidental exposures $(98.8 \%)$ and a male predominance $(59.7 \%)$ in children. Categories of poisoning exposures across all age groups were non-drug chemicals (52.7\%), medicines (35.2\%) and biological toxins (12.6\%). Pesticides (34.8\%), irritant/corrosive substances (27.7\%) and volatile hydrocarbons $(8.3 \%)$ were the most common classes of non-drug chemical exposures. Cholinesterase inhibitors (8.8\%), anticoagulant rodenticides $(7.1 \%)$ and pyrethroids $(5.0 \%)$ were the most commonly ingested non-drug chemicals. Aldicarb and amitraz poisoning were identified as toxicovigilance targets. Analgesics $(26.1 \%)$ were the most common class of medicine-related exposure, and paracetamol (15.8\%), benzodiazepines $(9.2 \%)$ and antihistamines $(5.2 \%)$ were the most common medicine-related exposures.

Conclusion. The study provided information on evolving trends and identified toxicovigilance targets and the need for continuing toxicology education programmes.
\end{abstract}

S Afr Med J 2013;103(5):293-297. DOI:10.7196/SAMJ.6647

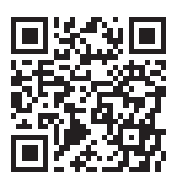

One of the greatest obstacles to effective prevention and control of poisoning is the lack of reliable epidemiological data. ${ }^{[1]}$ Poisoning data can be derived from many different sources, such as hospital records and poison information centre (PIC) records. However, each of these sources is limited by inherent biases. ${ }^{[2]}$ The actual incidence and spectrum of acute poisonings in South Africa (SA) are unknown, and there have been no publications on PIC data across all age groups in 10 years and none at all on combined hospital and PIC data across all age groups.

PICs have a fundamental role in toxicovigilance, which is defined by the World Health Organization as the active observation and evaluation of toxic risks and phenomena in the community - an activity that should result in measures aimed to reduce or remove risks. ${ }^{[3]}$ PIC data provide a unique perspective of poisoning, as not all exposures to poisonous substances result in hospital admissions and fatalities. PIC data include data on the type of poisoning information most often requested by medical professionals and also information requested by the general public. Data collected by a PIC that provides a national service, 24 hours a day and 7 days a week, afford this perspective on a national scale and serve as a valuable guide to trends in poisoning and moreover to the design and implementation of continuing education programmes in toxicology, at a community level as well as for medical professionals.

In contrast, data on actual hospital admissions that are underreported by PICs would be expected to provide additional information such as the actual incidence of admissions for acute poisoning as well as morbidity and mortality rates. As these would be expected to be the more severe cases of poisoning, knowledge of interventions that might have resulted in a more favourable outcome if implemented would also be of benefit.

A toxicovigilance survey was therefore designed, firstly to examine data from all telephonic consultations with the Tygerberg PIC (TPIC) (this paper) and then, separately, to examine hospital admissions data from the adjoining Tygerberg Academic Hospital (TAH) and all hospital-based calls made to the TPIC (the following paper). ${ }^{[4]}$ The objective was to collect current epidemiological data on poisoning in SA from these two different sources to investigate evolving trends in poisoning in the country. This paper reports the data surveyed from all telephonic consultations with the TPIC for a period of a year, with the objective of establishing the extent of the problem.

\section{Methods}

The Tygerberg PIC and the adjoining 1 310-bed TAH are situated in Parow, Cape Town. The TPIC handles more calls annually on a national basis than the other two national PICs combined and is the only PIC offering a consultant-based service, 24 hours a day and 7 days a week, to all South Africans. A study of the incidence of poisoning in SA as recorded by the TPIC was therefore considered suitable to provide meaningful data for the survey.

TPIC data based on telephonic consultations for a period of 1 year (1 August 2008 - 31 July 2009) were extracted from standard consultation forms and analysed for patient demographics, type of poisonous exposure, time from exposure to call $(<2$ hours or 
$>2$ hours), presenting symptoms and signs, gastric decontamination methods, interlocutor details and geographical distribution of calls. Cases of poisoning in animals were excluded.

The severity of exposure at the time of the call was graded using the Poison Severity Score (PSS), where $0=$ none (no symptoms or signs related to poisoning); $1=$ minor (mild, transient and spontaneously resolving symptoms); 2 = moderate (pronounced or prolonged symptoms); and $3=$ severe or life-threatening symptoms. ${ }^{[5]}$

'Children' were defined as paediatric patients $<13$ years old. Bleaches, powdered detergents, toilet cleaners, potassium permanganate, and corrosive alkalis and acids were grouped together and classified as 'irritant/corrosives'. Exposures to organophosphates and carbamates were grouped together and classified as 'cholinesterase inhibitors' The term 'intentional exposure' was used instead of 'deliberate selfpoisoning' for the sake of brevity. Exposures to biological toxins and medication errors were classified as accidental exposures. Ethanol as a co-ingested beverage was classified as a non-drug chemical ${ }^{[6]}$ All data were committed to a standard MS Excel spreadsheet and data analysis was performed using descriptive statistics.

\section{Results}

The TPIC dealt with 5538 calls during the study period, of which $42 \%$ were from the Western Cape province and the remainder from elsewhere in SA. Of these calls, 4771 were consultations related to exposures to poisonous substances. Hospital-based consultations comprised 2459 (51.5\%) of these calls, the remainder being queries from general practitioners $(874,18.3 \%)$, allied health professionals (207; 4.3\%) and the general public (1 231;25.8\%).

Demographic data on age and gender distribution are illustrated in Table 1 and the reasons for exposure in Table 2. Across all age groups, accidental poisoning was more common than intentional poisoning $(65.2 \%$ v. $34.8 \%)$. Of the cases, $55.8 \%$ were adults, of which $61.4 \%$ were intentional exposures; of these cases, $64.3 \%$ were females. Accidental poisoning was predominant in children (98.8\%), and intentional poisoning occurring in 25 cases, was seen only in the 5 - 12-year-old group. The male/female ratio of all cases was 1:1.4 in adults and 1.5:1 in children. Of the children, $87.2 \%$ were $<5$ years old.

As illustrated in Table 3, categories of exposures to poisonous substances across all age groups were non-drug chemicals (52.7\%) medicines (35.2\%), and biological toxins (spiders $>$ plants $>$ snakes $>$ scorpions) (12.6\%). Paracetamol (15.8\%), benzodiazepines $(9.2 \%)$ and antihistamines (5.2\%) were the most common medicine-related exposures, and $13.8 \%$ of cases were multiple drug overdoses (Table 4). Medicines used for pain control comprised $26.1 \%$ of cases, and of these medicines $28.3 \%$ were combination analgesic preparations. In children, $29.5 \%$ of cases were medicine-related, of which $86.4 \%$ were in the age group $<5$ years (Table 4 ). Ingestion was the most common route of exposure.

As shown in Table 5, the most common groups of non-drug chemical exposures across all age groups were pesticides (34.8\%), irritant/corrosives (27.7\%), and volatile hydrocarbons (8.3\%); 61.9\% of cases in children aged $<5$ years were related to exposures to non-drug chemicals. In this age group, $21.5 \%$ of exposures (245) were to household cleaning products and $2.5 \%$ to paraffin. The most common pesticide exposures across all age groups were cholinesterase inhibitors (25.3\%), anticoagulant rodenticides $(20.5 \%)$ and pyrethroids (14.4\%). No cases of occupational exposure to agricultural pesticides were reported. Aldicarb was ingested as a 'rat poison' in $17.2 \%$ of the cholinesterase inhibitor cases, and in $81.6 \%$

Table 1. Demographic data from the Tygerberg PIC study: Age and gender distribution of cases

\begin{tabular}{llll}
\hline & Total cases (column \%) & Male (row \%) & Female (row \%) \\
\hline Children $<5$ years & $1839(38.5)$ & $1095(59.5)$ & $744(40.5)$ \\
Children 5 - 12 years & $271(5.7)$ & $164(60.5)$ & $107(39.5)$ \\
All children <13 years & $2110(44.2)$ & $1259(59.7)$ & $851(40.3)$ \\
Adults & $2661(55.8)$ & $1129(42.4)$ & $1532(57.6)$ \\
Total cases (row \%) & 4771 & $2388(50.1)$ & $2383(49.9)$
\end{tabular}

Table 2. Demographic data from the Tygerberg PIC study: Reason for exposure and distribution by age and gender

\begin{tabular}{llll}
\hline & Total cases (column \%) & Male (row \%) & Female (row \%) \\
\hline $\begin{array}{lll}\text { Intentional exposures (deliberate self-poisoning) } \\
\text { Children }<5 \text { years }\end{array}$ & 0 & 0 & 0 \\
Children $5-12$ years & $25(1.5)$ & $10(40.0)$ & $15(60.0)$ \\
Adults & $1635(98.5)$ & $584(35.7)$ & $1051(64.3)$ \\
$\quad$ Total & $1660(34.8)$ & $594(35.8)$ & $1066(64.2)$ \\
Accidental exposures & & $1097(59.7)$ & $742(40.3)$ \\
Children $<5$ years & $1839(59.1)$ & $154(62.6)$ & $92(37.4)$ \\
Children 5 - 12 years & $246(7.9)$ & $551(53.7)$ & $475(46.3)$ \\
Adults & $1026(33.0)$ & $1802(57.9)$ & $1309(42.1)$ \\
$\quad$ Total & $3111(65.2)$ & 2388 & 2383
\end{tabular}


Table 3. Demographic data from the Tygerberg PIC study: Category and type of toxic exposure and route of exposure

\begin{tabular}{ll}
\hline & $\begin{array}{l}\text { Total cases } \\
\text { (column \%) }\end{array}$ \\
\hline Exposures to medicines (pharmaceuticals) & \\
Children $<5$ years & $538(32.0)$ \\
Children 5 - 12 years & $85(5.1)$ \\
Adults & $1058(62.9)$ \\
$\quad$ Total & $1681(35.2)$ \\
Exposures to non-drug chemicals & \\
Children $<5$ years & $1139(45.3)$ \\
Children 5 - 12 years & $107(4.3)$ \\
Adults & $1266(50.4)$ \\
$\quad$ Total & $2512(52.7)$ \\
Exposures to biological toxins & \\
Children $<5$ years & $147(24.4)$ \\
Children 5 - 12 years & $65(10.8)$ \\
Adults & $390(64.8)$ \\
Snakebite & $86(14.3)$ \\
Spider bite & $130(21.6)$ \\
Scorpion sting & $59(9.8)$ \\
Plants & $96(15.9)$ \\
Exposures to other biological toxins & $231(38.4)$ \\
Total & $602(12.6)$ \\
Most common route of exposure & \\
Ingestion & $3895(81.6 \%$ of all \\
Total cases in study & cases $)$ \\
& 4771 \\
&
\end{tabular}

(31) of these the TPIC assisted in its identification as a carbamate and not an anticoagulant. Poisoning with amitraz (variously reported as a tick, cattle, cow, sheep or dog dip or 'unknown pesticide') was identified by the TPIC in $89.1 \%$ ( 41 of the 46 ) cases. In all instances the callers were unfamiliar with amitraz and its potential to cause lifethreatening poisoning, and in $21.7 \%$ of these cases the poisoning had been misdiagnosed as organophosphate poisoning.

In $20.5 \%$ of cases (980) the call was made to the PIC within 2 hours of the toxic exposure. With regard to the severity of poisoning reported at the time of the call, $74.8 \%$ of patients (3 568) were asymptomatic (PSS 0). Vomiting was the most common presenting symptom $(5.5 \%, 264)$. Severity of the exposure was rated as PSS 1 in $15.7 \%$ of cases (749), PSS 2 in 5.3\% (251) and PSS 3 in 4\% (190). There were 11 deaths, all of adults, and the majority $(8,72.7 \%)$ of these were due to pesticide exposures.

\section{Discussion}

Published studies on the epidemiology of poisoning are based mainly on PIC records and hospital admissions data, as the most reliable sources of information. ${ }^{[2]}$ Data accumulated by PICs are routinely used by some governmental bodies to set policies and direct funding. However, as calls to the PICs by hospital staff are voluntary and spontaneous in most cases, underutilisation of the facility results in discrepancies in epidemiological estimates as a result of incomplete reporting/under-reporting of the more common poisonings by hospitals to PICs. ${ }^{[7]}$ This survey has illustrated a similar lack of concordance in SA, where calls to a PIC are not compulsory. During the period reviewed, the TPIC was consulted on the identification and management of the poisoning in only $7.9 \%$ of Tygerberg hospital admissions for acute poisoning. This underutilisation of an associated PIC, resulting in under-reporting, has been noted elsewhere. ${ }^{[]]}$Trends identified in this study were used by the PIC to direct further toxicovigilance and education efforts to the member hospital, health professionals and the general public.

This study has shown that accidental poisoning was reported to the TPIC more commonly than intentional poisoning ( $65.2 \%$ v. $34.8 \%)$, and that there was a female predominance in adult poisonings (57.6\%) and a male predominance in childhood poisonings (59.7\%). This distribution is similar to that found in a major national PIC study undertaken in the USA. ${ }^{[6]}$ However, unlike the US study, our study revealed that poisonous exposures were more common in adults than children $(55.8 \%$ v. $44.2 \%)$ and that there were more exposures to non-drug chemicals than to medicines $(52.7 \% \mathrm{v}$. $35.2 \%$ ) across all age groups. ${ }^{[6]}$ Also, as shown in other European studies, intentional poisoning was more common in adults, and predominantly in females (male/female ratio 1:1.8). ${ }^{[8,9]}$

Poisoning in young children is preventable, and it is well known that age is an important factor in childhood poisoning. Infants and children under the age of 5 are physically closer to the ground than older children, and items such as rat poisons and other pesticides and household chemicals, unsafely stored in easily accessible kitchen and bathroom cupboards, are easily visible and tempting. The natural curiosity of young children leads them to put their hands and small objects into their mouths. ${ }^{[1]}$ Studies have shown that non-fatal poisoning is most common in the 1 - 4-year age group. ${ }^{[1,6]}$ In our survey, $87.2 \%$ of children were younger than 5 years, and in all these cases the poisoning was accidental. In this age group, as shown in other studies, non-drug chemicals were responsible for the majority of the exposures, with boys more prone to accidental exposure to poisonous substances than girls. ${ }^{[6]}$ Reports of paraffin ingestion were few in this group of patients $(2.5 \%)$, possibly because these children were taken directly to hospital rather than after consultation with the PIC and because health professionals are familiar with the management of this type of poisoning. The dangers of paraffin poisoning in SA children are well documented and community education drives by the Paraffin Safety Association of SA ensure that the exposure is known to be potentially lethal.

The incidence of non-drug-related poisoning in our study was found to be higher than that of medicine-related poisoning. As shown in a Zimbabwean study, pesticides were the most common group of nondrug-related exposures and the most common group of all exposures in the study $(18.3 \%) .{ }^{[10]}$ Cholinesterase inhibitors (4.6\% of cases), anticoagulant rodenticides and pyrethroids were the most frequent pesticide exposures. In contrast to the Zimbabwean experience, where $10 \%$ of accidental exposures to pesticide toxicity were occupational exposures, ${ }^{[10]}$ there were no such cases in this study. Of the cholinesterase inhibitor exposures $17.2 \%$ were due to ingestion of the carbamate pesticide aldicarb, sold as 'rat poison'. It is variously named 'rat poison', 'rattex', 'two step', 'galephirime' or 'halethrini'. Aldicarb is sold illegally as a 'street pesticide' via unlicensed outlets and street vendors in the form of black granules or powder (described as 'poppy seeds', 'black pepper' or 'black powder') in unlabelled plastic sachets, typically cylindrical in shape (approximately $10 \mathrm{~mm} \times 45 \mathrm{~mm}$ ) and called 'straws'. Aldicarb is sold legally in SA for restricted agricultural use only, as Temik. The illegal sale of unregistered, unlabelled 'street pesticides' such as aldicarb is an emerging problem in SA as it is in other developing countries. ${ }^{[10-12]}$ It is also used in SA, and elsewhere, by criminals to maliciously poison 


\section{RESEARCH}

Table 4. Most common medicine-related exposures ${ }^{\star}$

\begin{tabular}{llll}
\hline Medicines (pharmaceuticals) & Total cases (column \%) & Adults (column \%) & Children <13 years (column \%) \\
\hline Analgesics & $438(26.1)$ & $389(36.8)$ & $49(7.9)$ \\
Paracetamol & $266(15.8)$ & $234(22.1)$ & $32(5.1)$ \\
NSAIDs & $70(4.2)$ & $53(5.1)$ & $17(2.7)$ \\
Codeine & $49(2.9)$ & $49(4.6)$ & 0 \\
Aspirin & $34(2.0)$ & $34(3.2)$ & 0 \\
Dextropropoxyphene & $14(0.8)$ & $14(1.3)$ & 0 \\
Tramadol & $5(0.3)$ & $5(0.5)$ & 0 \\
Combination analgesics & $124(7.4)$ & $124(11.7)$ & 0 \\
Benzodiazepines & $155(9.2)$ & $155(14.6)$ & 0 \\
Antihistamines & $88(5.2)$ & $48(4.5)$ & $40(6.4)$ \\
SSRIs & $77(4.6)$ & $77(7.3)$ & 0 \\
Carbamazepine & $60(3.6)$ & $48(4.5)$ & $12(1.9)$ \\
Amitriptyline & $51(3.0)$ & $44(4.2)$ & $7(1.1)$ \\
Theophylline & $25(1.5)$ & $20(1.9)$ & $5(1.6)$ \\
Phenytoin & $14(0.8)$ & $14(1.3)$ & 0 \\
Drugs of abuse & 0 & 0 & 0 \\
Multiple drug ingestions & $232(13.8)$ & $216(20.4)$ & $16(2.6)$ \\
Total number of medicine-related exposure cases & $1681(35.2)$ & $1058(39.8)$ & $623(29.5)$ \\
Total cases in study & 4771 & 2661 & 2110 \\
NSAIDs = non-steroidal anti-inflammatory drugs; SSRIs = selective serotonin reuptake inhibitors. & & \\
*The individual potentially toxic constituent agents in fixed-dose combination drug formulations were recorded as separate agents. For this reason, and because more than one type of agent was \\
often co-ingested, the sum of percentages may be represented as $>100 \%$ in certain cases.
\end{tabular}

Table 5. Most common non-drug chemical (non-pharmaceutical) exposures

\begin{tabular}{|c|c|c|c|c|}
\hline Non-drug chemical & $\begin{array}{l}\text { Total cases } \\
\text { (column \%) }\end{array}$ & $\begin{array}{l}\text { Adults } \\
\text { (column \%) }\end{array}$ & $\begin{array}{l}\text { Children } 5 \text { - } 12 \text { years } \\
\text { (column \%) }\end{array}$ & $\begin{array}{l}\text { Children }<5 \text { years } \\
\text { (column \%) }\end{array}$ \\
\hline Pesticides $^{*+}$ & $874(34.8)$ & $519(41.0)$ & $48(44.9)$ & $307(27.0)$ \\
\hline Cholinesterase inhibitors (organophosphates and carbamates) & $221(8.8)$ & $160(12.6)$ & $12(11.2)$ & $49(4.3)$ \\
\hline Anticoagulant rodenticides & $179(7.1)$ & $75(5.9)$ & $10(9.3)$ & $94(8.3)$ \\
\hline Pyrethroids & $126(5.0)$ & $76(6.0)$ & $6(5.6)$ & $44(3.9)$ \\
\hline Amitraz & $46(1.8)$ & $27(2.1)$ & $4(3.7)$ & $15(1.3)$ \\
\hline Organochlorines & $39(1.6)$ & $31(2.4)$ & $2(1.9)$ & $6(0.5)$ \\
\hline Napthalene & $33(1.3)$ & $3(0.2)$ & $6(5.6)$ & $24(2.1)$ \\
\hline Glyphosate & $23(0.9)$ & $23(1.8)$ & 0 & 0 \\
\hline Paraquat & $15(0.6)$ & $14(1.1)$ & 0 & $1(0.1)$ \\
\hline Aluminium phosphide & $8(0.3)$ & $7(0.6)$ & 0 & $1(0.1)$ \\
\hline Borax & $7(0.3)$ & 0 & $3(2.8)$ & $4(0.4)$ \\
\hline Other pesticides & $177(7.0)$ & $103(8.1)$ & $5(4.7)$ & $69(6.1)$ \\
\hline Aldicarb 'rat poison' (included in cholinesterase inhibitors) & $38(1.5)$ & $30(2.4)$ & 0 & $8(0.7)$ \\
\hline Irritant/corrosives & $695(27.7)$ & $374(29.5)$ & $23(21.5)$ & $298(26.2)$ \\
\hline Volatile hydrocarbons (including paraffin) & $209(8.3)$ & $97(7.7)$ & $1(0.9)$ & $111(9.7)$ \\
\hline Paraffin & $41(1.6)$ & $12(0.9)$ & 0 & $29(2.5)$ \\
\hline Ethanol as a co-ingested beverage & $64(2.5)$ & $64(5.1)$ & 0 & 0 \\
\hline Total cases of non-drug chemical-related exposures & $2512(52.7)$ & $1266(53.0)$ & $107(39.5)$ & $1139(61.9)$ \\
\hline Total cases in study & 4771 & 2388 & 271 & 1839 \\
\hline
\end{tabular}


dogs in order to gain access to residential premises. ${ }^{[13]}$ Aldicarb was identified as a toxicovigilance target by TPIC consultants. By careful questioning, many of these cases reported as 'rat poison' ingestions were identified and clinicians were advised on the appropriate management of the exposures as cholinesterase inhibitor toxicity and not the anticipated toxicity of the long-acting anticoagulants. Another source of confusion in the management of pesticide exposures is amitraz, a novel formamidine pesticide used frequently as an antitick dip in SA. ${ }^{[14]}$ The TPIC consultants were able to guide healthcare professionals on the identification and management of these exposures, often misdiagnosed and treated as organophosphate poisonings owing to similar clinical features on presentation. ${ }^{[14]}$

As has been shown elsewhere, analgesic poisoning was the most common class of medicine-related exposure and comprised 9.2\% of total exposures in the study. ${ }^{[6,9,9]}$ Paracetamol was the single most common exposure across all age groups (5.6\% of all exposures). Paracetamol is a non-prescription drug in SA and therefore easily accessible. The incidence of ingestion of the more highly scheduled opioid analgesics (dextropropoxyphene and tramadol) was negligible. It should be noted that although much media attention has been given to the potential toxicity of dextropropoxyphene in overdose, and although it has recently been withdrawn from the market in SA, this study revealed that dextropropoxyphene was very seldom used for intentional overdose and no fatalities were reported. ${ }^{[15]}$ What probably played a role in preventing its use for the purpose of selfharm was that this was a highly scheduled drug in SA, available on prescription only, and that formulations were expensive.

A matter of concern is the fact that the most common exposures in this study, i.e. paracetamol and household pesticides such as anticoagulant rodenticides, pyrethroids, some cholinesterase inhibitors and irritant/corrosive cleaning agents, are readily accessible on supermarket shelves in SA. Should these not be sold, as cigarettes and alcohol are, in restricted areas of the supermarkets to make the general public aware of their potential toxicity?

This study has several limitations associated with the use of PIC data. As these data are restricted to voluntary calls, they reflect only information provided when the public or healthcare professionals report an exposure to a poisonous substance to the PIC. There is a distinction between enquiries related to exposures to potentially toxic substances and acute poisonings, as the one does not necessarily lead to the other ${ }^{[1,2]}$ The spectrum of consultations dealt with by the PIC may be a reflection of the needs of healthcare professionals rather than an indication of the actual national incidence of poisoning. Under-reporting is possible owing to lack of awareness of the accessibility and functions of the PIC as well as to language barriers or difficulty with calls from remote rural areas. ${ }^{[2]} \mathrm{A}$ contradictory limitation to using PIC data is possible over-reporting of the frequency of poisonings that are rare or unfamiliar. PSS scoring in this PIC study should be interpreted with caution, as this is based on information provided by the enquirer/interlocutor. ${ }^{[16]}$

Conclusion The data retrieved from Tygerberg PIC consultations have provided important information on the incidence and spectrum of poisoning in SA. The most common findings across all age groups were that: $(i)$ accidental poisoning was more common than intentional poisoning; (ii) the incidence of non-drug chemical exposures was higher than that of medicine-related exposures; and (iii) pesticides, particularly cholinesterase inhibitors, were the most common non-drug chemical exposures, and (iv) analgesics, particularly paracetamol, were the most common medicinerelated exposures. Other findings were the predominance of females in adult exposures and of intentional poisoning in adults (again predominantly among females), and male predominance in children, with chiefly accidental exposures and exposures to nondrug chemicals.

A comprehensive comparison of the results of this study with other similar published studies is limited by the fact that the true incidence of acute poisoning globally is uncertain, and severely restricted by the paucity of such studies from the rest of Africa. However, the high incidence of cholinesterase inhibitor and paracetamol exposures, age and gender demographics in children and female predominance in the adult groups do reflect similar distributions when compared with international and African reports. ${ }^{[6,8-12]}$

Of greatest concern is the fact that the most common agents responsible for poisoning in this study are readily available on supermarket shelves in SA. Exposures to aldicarb 'rat poison' and amitraz were identified as ongoing toxicovigilance targets for the TPIC.

Although PIC data are not necessarily a true reflection of the incidence and spectrum of poisoning, these data sources indicate the extent of the problem and provide guidance in devising strategies to reduce mortality and morbidity and in the design and implementation of continuing toxicology education programmes offered to the community and to medical professionals.

Acknowledgements. Our sincere thanks to all the staff of the Tygerberg Poison Information Centre for their valuable contribution to this study.

Conflict of interest. The authors report no conflicts of interest. The authors alone are responsible for the content and writing of the paper.

Ethics approval. Ethics approval No. NO8/06/71 from the Stellenbosch University Committee for Human Research.

\section{References}

1. Meredith TJ. Epidemiology of poisoning. Pharmacol Ther 1993;59:251-256.

Hoffman RS. Poison information centers and poison epidemiology In: Flomenbaum NE, Goldfrank LR Hoffman RS, Howland MA, Lewin N, Nelson L, eds. Goldfrank's Toxicologic Emergencies. 8th ed. New
Hellan York: McGraw-Hill, 2006:1826-1828.

3. Guidelines for Poison Control. http://www.who.int/ipcs/publications/training_poisons/guidelines poison_control/en/index5.html (accessed 6 July 2012).

4. Veale DJH, Wium CA, Müller GJ. Toxicovigilance II: A survey of the spectrum of acute poisoning and current practices in the initial management of poisoning cases admitted to South African hospitals. S Afr Med J 2013;103(5):298-303. [http://dx.doi.org/10.7196/SAMJ.6648]

5. Persson H, Sjöberg GK, Haines IA, Pronkzuk de Garbino J. Poisoning Severity Score. Grade of acute poisoning. J Toxicol Clin Toxicol 1998;36(3):205-213. [http://dx.doi.org/10.3109/15563659809028940]. 6. Watson WA, Litovitz TL, Rodgers GC, et al. 2004 Annual Report of the American Association of Poison Control Centers Toxic Exposure Surveillance System. Am J Emerg Med 2005;23:589-666. [http://dx.doi. org/10.1016/j.ajem.2005.05.001].

7. Harchelroad F, Clark RF, Dean B, Krenzelok EP. Treated vs reported toxic exposures: discrepancies between a poison control center and a member hospital. Veterinary \& Human Toxicology 1990;32(2):156-159.

8. Prescott K, Stratton R, Freyer A, Hall I, Le Jeune I. Detailed analysis of self-poisoning episodes presenting to a large regional teaching hospital in the UK. Br J Clin Pharmacol 2009;68(2):260-268. [http://dx.doi. org/10.1111/j.1365-2125.2009.03458.x]

. Doak MW, Nixon AC, Lupton DJ, Waring WS. Self-poisoning in older adults: patterns of drug ingestion and clinical outcomes. Ageing 2009;38:407-411. [http://dx.doi.org/10.1093/ageing/afp046]

10. Tagwireyi D, Ball D, Nhachi C. Toxicoepidemiology in Zimbabwe: pesticide poisoning admissions to major hospitals. Clin Toxicol 2006;44(1):59-66. [http://dx.doi.org/10.1080/15563650500394878]

11. Balme KH, Roberts JC, Glasstone M, et al. Pesticide poisonings at a tertiary children's hospital in South Africa: an increasing problem. Clin Toxicol 2010;48(9):928-934. [http://dx.doi.org/10.3109/15563650.2 010.534482]

12. Favara DM. The burden of deliberate self harm on the critical care unit of a peri-urban referral hospital in the Eastern Cape: a five year review of 419 patients. S Afr Med J 2013;103(1):40-43. [http://dx.doi. org/10.7196/SAMJ.6231]

13. Arnot LF. Veale DJH, Steyl JC, Myburgh JG. Treatment rationale for dogs poisoned with aldicarb (carbamate pesticide). J S Afr Vet Assoc 2011;28(4):232-238. [http://dx.doi.org/10.4102/jsava.v82i4.80] 4. Veale DJH, Wium CA, Muller GJ. Amitraz poisoning in South Africa - a two year survey (2008-2009). Clin Toxicol 2011;49(1):40-44. [http://dx.doi.org/10.3109/15563650.2010.542159]

. Beeton A. Dextropropoxyphene is dead? Long live.... Southern African Journal of Anaesthesia and Analgesia 2011;17(4):266-280

16. Adams RD, Gibson AL, Good AM, Bateman DN. Systematic differences between healthcare professionals and poison information staff in the severity scoring of pesticide exposures. Clin Toxicol 2010;48(6):550-558. [http://dx.doi.org/10.3109/15563650.2010.491484] 